\title{
Spin polarization in photoemission from the cuprate superconductor $\mathrm{Bi}_{2} \mathrm{Sr}_{2} \mathrm{CaCu}_{2} \mathrm{O}_{8+\delta}$
}

\author{
Mauro Fanciulli, ${ }^{*}$ Stefan Muff, Andrew P. Weber, and J. Hugo Dil \\ Institut de Physique, École Polytechnique Fédérale de Lausanne, CH-1015 Lausanne, Switzerland \\ and Swiss Light Source, Paul Scherrer Institut, CH-5232 Villigen, Switzerland \\ (Received 5 April 2017; revised manuscript received 26 May 2017; published 20 June 2017)
}

\begin{abstract}
Photoelectrons produced from the excitation of spin-degenerate states in solids can have a sizable spin polarization, which is related to the phase of interfering channels in the photoemission matrix elements. Such spin polarization can be measured by spin-resolved photoemission spectroscopy to gain information about the transitions and the Wigner time delay of the process. Incorporating strongly correlated electron systems into this paradigm could yield a novel means of extracting phase information crucial to understanding the mechanism of their emergent behavior. In this work, we present, as a case study, experimental measurements of the cuprate superconductor $\mathrm{Bi}_{2} \mathrm{Sr}_{2} \mathrm{CaCu}_{2} \mathrm{O}_{8+\delta}$ by spin-resolved photoemission while maintaining full angular and energy resolution. A spin polarization of at least $10 \%$ is observed, which is related to the phase of the photoelectron wave function.
\end{abstract}

DOI: 10.1103/PhysRevB.95.245125

The electronic properties of crystals are best understood when information on their band structure can be obtained. Angle-resolved photoemission spectroscopy (ARPES) provides a direct means of reconstructing the band structure by measuring the energies and momenta of electrons photoexcited from a solid [1]. Spin- and angle-resolved photoemission spectroscopy (SARPES), in addition, measures the spin polarization $\boldsymbol{P}$ of the photoelectron beam as a function of emission angle and kinetic energy. This can show a net polarization if the initial state is polarized as, for example, in ferromagnets $[2,3]$ or spin-momentum-locked systems, such as Rashba-like materials [4-6] and topological insulators [7-10], but also as a result of the photoemission process as a whole [11]. If the incident light is circularly polarized, i.e., it carries angular momentum, the spin polarization of the photoelectron beam from solid-state targets can, in several different circumstances, be interpreted as a result of the selection rules for the considered transitions along the lines of the atomic Fano effect [12-16].

Also, when the incident light is linearly polarized or unpolarized, i.e., it does not transfer a net angular momentum, the photoelectron beam can still present a sizable $\boldsymbol{P}$. The origin of this effect lies in the symmetry reduction of angledependent photoemission and in the interference of different photoemission channels. The size of $\boldsymbol{P}$ is related to the phase shift of the complex matrix elements describing the transitions under consideration [17-19]. One can, therefore, access the phase information by measuring the spin polarization of the photoelectrons. In particular, a novel application of SARPES is the estimate of the Eisenbud-Wigner-Smith (EWS) time delay [20] $\tau_{\text {Ews }}$ of photoemission, defined as the derivative of the phase shift with respect to the photoelectron kinetic energy, obtained from the measured $\boldsymbol{P}$, as shown in Ref. [19] for the model system $\mathrm{Cu}(111)$. By expressing the spin polarization as a function of the radial parts and phase shifts of the matrix elements, along the lines of the atomic half-scattering formalism [18], one can analytically obtain an estimate for a finite inferior limit to the EWS time delay in the attosecond

\footnotetext{
*Corresponding author: mauro.fanciulli@epfl.ch
}

domain. In particular, under the assumption that the moduli of the matrix elements do not vary in the considered $E_{b}$ range, by measuring the slope of spin polarization versus electron binding energy $E_{b}$, one can estimate $\left|\tau_{\mathrm{Ews}}\right|>\hbar\left|d P / d E_{b}\right|$. A better knowledge of the matrix elements and a detailed description of the scattering geometry can lead to an improved estimate. This approach is complementary to attosecondresolved spectroscopies, such as photoemission streaking [21] and reconstruction of attosecond beating by interference of two-photon transitions (RABBITT) [22], and, by combining them with SARPES, one could tackle the fundamental issue of the chronoscopy of photoemission [23].

From the materials point of view, the phase information can also be employed in order to better understand correlations because phase is vital to the full description of the electronic structure in strongly correlated electron systems. In this paper, we present experimental results on the optimally doped cuprate $\mathrm{Bi}_{2} \mathrm{Sr}_{2} \mathrm{CaCu}_{2} \mathrm{O}_{8+\delta}$ (BSCCO 2212, $T_{C} \approx 95 \mathrm{~K}$ ), measured by spin-resolved photoemission while maintaining the full energy and momentum resolution of ARPES. Cuprate superconductors are representative systems for the study of electron correlations and have been probed extensively by ARPES [24-29]. Furthermore, it has already been shown in a previous work [30] that spin-resolved angle-integrated resonant photoemission can help improve the description of their electronic structure by determining, for instance, the Zhang-Rice singlet character of the relevant low-energy states in BSCCO. We show that even in the less-specific case of off-resonance photoemission, an energy- and angle-resolved $\boldsymbol{P}$ is measured, thus allowing the access to the phase information. These results are therefore a case study that will open the way for further experiments and lead to qualitatively new insights in the study of strong correlations in high-temperature superconductors.

The experiments were performed at the COPHEE endstation at the Swiss Light Source [31,32]. This endstation is equipped with an Omicron EA125 hemispherical analyzer and two orthogonally placed classical Mott detectors. The relevant experimental geometry is shown in Fig. 1(a). Because of the low photoemission count rate of the states close to the Fermi level and the need to mitigate effects of deterioration of the 

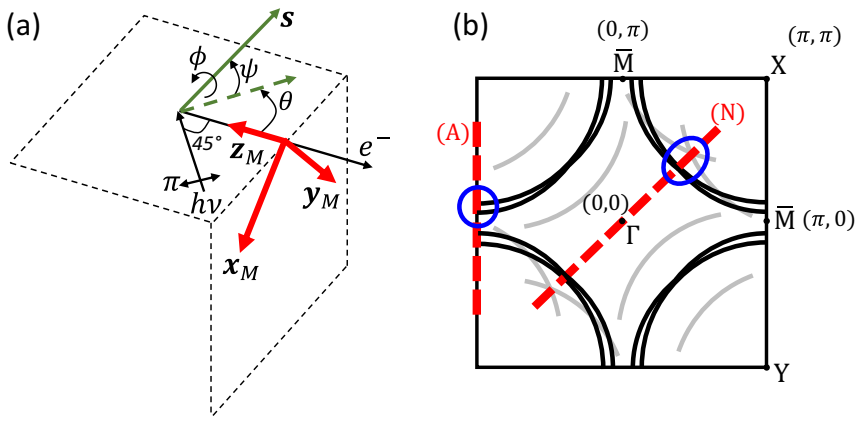

FIG. 1. (a) Definition of relevant geometry in the experimental setup of the COPHEE endstation. In order to access different points of the reciprocal space, the sample surface normal $s$ is rotated by $\theta$ from the electron-detection direction and by $\psi$ from the scattering plane. The crystal orientation is changed by the azimuthal angle $\phi$. The $\pi$ polarized photon and the electron directions are fixed. The Mott spin detector coordinate frame $\left(x_{M}, y_{M}, z_{M}\right)$ is also shown. (b) Scheme of the Fermi surface of BSCCO 2212. The red lines (N) and $(\mathrm{A})$ indicate the nodal and antinodal directions, respectively, that have been probed with the spin-resolved MDCs of Figs. 2 and 3.

sample's surface with time, only one of the two Mott detectors of the COPHEE endstation was used. Thus only two spatial components of $\boldsymbol{P}$ were measured, namely, $x_{M}$ and $z_{M}$. The different regions of the Fermi surface were reached by moving the sample's normal $s$ by changing the angles $\phi, \theta$, and $\psi$.

The sample growth and characterization are reported in Ref. [33]. The band under consideration is the $\mathrm{CuO}_{2}$ derived state of BSCCO 2212, which is relevant for the superconductivity. This state, as shown in the Fermi surface in Fig. 1(b) with its common labeling, encloses the Brillouin zone corners. It presents a splitting into bonding and antibonding states due to the presence of a $\mathrm{CuO}_{2}$ bilayer within the unit cell [24] and is affected by umklapp bands due to diffraction by the $\mathrm{Bi}$ atoms at the cleaved surface [34]. All momentum distribution curves (MDCs) shown in Figs. 2 and 3 were measured by scanning the angle $\psi$ and using $\pi$ polarized light of energy $h v=50 \mathrm{eV}$. In particular, the nodal $(\mathrm{N})$ and antinodal (A) directions in the Brillouin zone were considered. However, due to the difficulties inherent in obtaining precise sample alignment with the channeltron-based setup at COPHEE, the alignment could be imperfect, as indicated by two blue circles on the nodal and antinodal directions in Fig. 1(b). The BSCCO samples were cleaved and measured in situ at low temperature $(T=20 \mathrm{~K})$ and at a base pressure lower than $1 \times 10^{-10} \mathrm{mbar}$ in order to obtain a clean surface for the SARPES experiments. The surface quality and orientation were checked by lowenergy electron diffraction. All measurements were performed at $20 \mathrm{~K}$.

In Figs. 2(a) and 2(b), the two $P_{x_{M}}$ and $P_{z_{M}}$ spatial components of $\boldsymbol{P}$ are shown for MDC scans along the nodal and antinodal directions, respectively. The $P_{z_{M}}$ component shows no clear dependence on momentum. On the other hand, all the bands at positive and negative momenta for the nodal and antinodal directions present a clear momentum-resolved spin polarization along the $x_{M}$ direction, with a value that reaches $10 \%$. Notably, each photoemission intensity peak in the antinodal direction presents an up-down spin structure reminiscent of the double polarization feature (DPF) observed in $\mathrm{Cu}(111)$ [19]. In the nodal direction, the photoemission intensity peaks are more structured, and there appear to be two partially overlapped DPFs, as can be better seen in Fig. 2(c), which shows a closeup of $P_{x_{M}}$ for negative momenta from Fig. 2(a). The signal was fitted with four Gaussian peaks, with the same width and opposite height for each pair of peaks constituting a DPF. Whereas the bonding and antibonding states might play a role in the spin signal but are not resolved, the two DPFs corresponding to the photoemission intensity peaks at $k_{\|} \approx-0.35 \AA^{-1}$ and $k_{\|} \approx-0.55 \AA^{-1}$ can be assigned to the main band and to the umklapp replica, respectively. As suggested by the structured photoemission intensity peak, the umklapp replica is possibly enhanced by the misalignment indicated by a blue circle in Fig. 1(b). A similar behavior is also observed at positive momenta, where, however, the total intensity is lower. It bears mentioning that the nature of the DPF, which seems to be a general property of dispersive bands whereas it does not appear in core levels [19], is not yet understood.

For comparison, the same scan along the nodal direction was measured in the nonsuperconducting state of the related compound BSCCO $2201\left(T_{C} \approx 8 \mathrm{~K}\right.$; see Ref. [35] for characterization), as shown in Fig. 2(d). The presence of a clear spin-polarization signal with DPF arising from this system as well, which has no bilayer splitting, excludes any possible interpretation of the spin-polarization signal as a direct consequence of the superconducting state, or bonding and antibonding splitting.

In Fig. 3(a), the band map measured on BSCCO 2212 along the nodal direction is shown. Red solid lines indicate the MDCs that were measured with spin resolution. Since the $P_{z_{M}}$ signal is considerably smaller compared to $P_{x_{M}}$, we consider only $P_{x_{M}}$. In order to determine the variation with $E_{b}$ to obtain the EWS time delay, we plot the value of the spin polarization for one peak from each MDC, choosing the one closer to $\Gamma$ because it is the cleanest by virtue of being less influenced by overlaps with other DPFs, as indicated by an arrow in Fig. 2(a). The plot of $P_{x}\left(E_{b}\right)$ is shown in Fig. 3(b), with an obtained absolute slope of $0.13 \mathrm{eV}^{-1}$. Since we have no information about the third component $P_{y_{M}}$, under the assumption that $\boldsymbol{P}$ does not vary direction but only its modulus in the measured $E_{b}$ range [19], we can write $d P / d E_{b}>d P_{x_{M}} / E_{b}$ and therefore find an inferior limit for the EWS time delay. If, for example, $P_{y_{M}} \approx 0$, we find $\tau_{\text {Ews }}>85$ as, whereas if $P_{y_{M}}\left(E_{b}\right) \approx P_{x_{M}}\left(E_{b}\right)$, then $\tau_{\text {Ews }}>120$ as. These values are at least three times larger than the one found for $\mathrm{Cu}(111)$ [19]. However, further experiments and theoretical modeling would be needed to determine whether this is due to the enhanced electron correlations in the copper-oxide plane. Furthermore, the investigated binding energy range was necessarily much smaller, and is therefore less suited to make precise estimates about the slope of $P\left(E_{b}\right)$.

Besides the estimate of $\tau_{\mathrm{EWS}}$, the main result of the present experiments on BSCCO is the presence itself of a significant spin polarization measured with energy and momentum resolution over the band close to the Fermi level. Such spin polarization is not an indication of a spin-polarized initial state, but is the result of the interference processes that build up the photoelectron wave function in the SARPES experiment. 

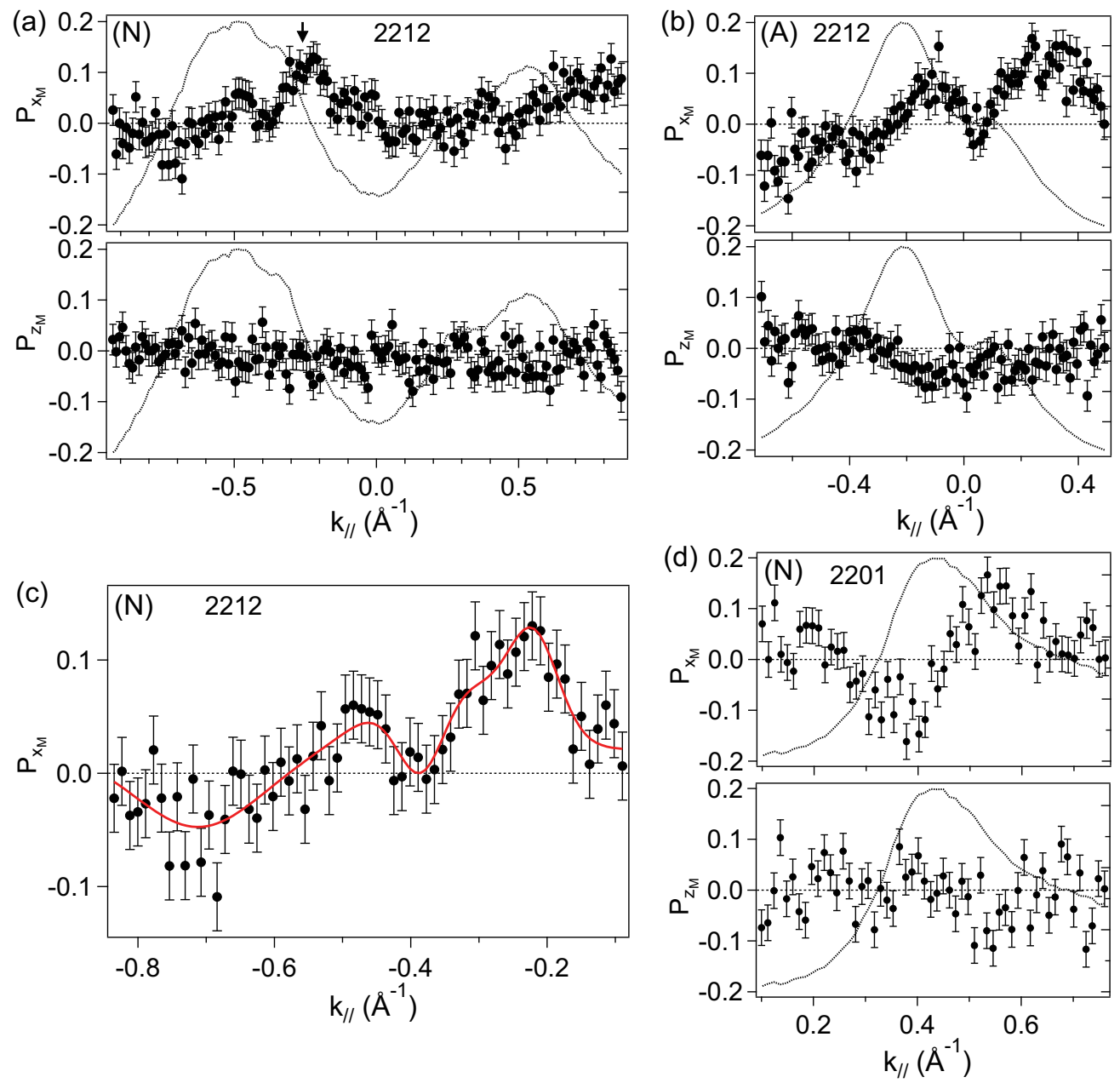

FIG. 2. $P_{x_{M}}$ and $P_{z_{M}}$ spatial components of $\boldsymbol{P}$ for MDCs along the (a) nodal and (b) antinodal directions at 40 meV below the Fermi level. The arrow in (a) indicates the momentum position of the spin-polarized peak considered to construct the plot of Fig. 3(b). (c) A closeup of $P_{x_{M}}$ for negative momenta from (a), in which two DPFs appear in the spin signal as highlighted by the fit with four Gaussian peaks (red line). (d) Reference measurement on BSCCO 2201, which is not superconducting at $20 \mathrm{~K}$; same direction as in (a). In (a), (b), (d), the photoemission total intensity is also shown in arbitrary units as a dotted black line.

This effect is expected to occur for every system in which the photoemission intensity is influenced by the interference of different channels, such as different orbital compositions, different projections of the light polarization, mixed double
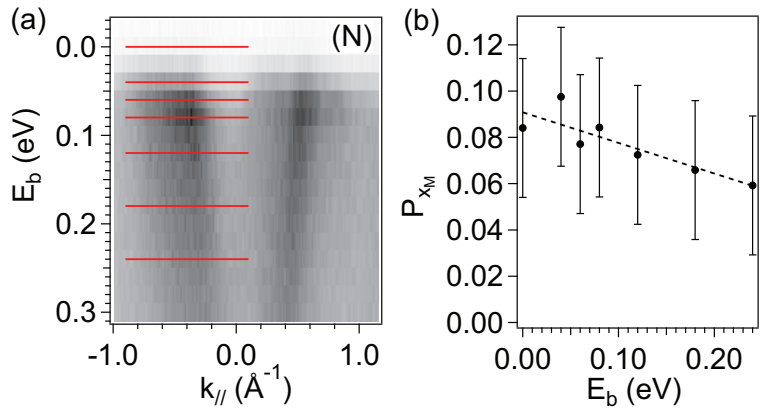

FIG. 3. (a) Band map along the nodal direction, with red lines indicating the MDCs measured with spin resolution; (b) plot of $P_{x_{M}}\left(E_{b}\right)$. The slope of $0.13 \mathrm{eV}^{-1}$ is used to estimate the EWS time delay. group symmetry configurations, and the overlap of different spin-degenerate initial or final states. Given the variety and complexity of these channels, the development of a general theory of photoelectron interference is needed to extend the application of this paradigm to different materials. In the particular case of BSCCO 2212, for example, the presence of bonding and antibonding states might as well play a role in defining the interfering transitions. The similar values of spin polarization measured on BSCCO 2201 above $T_{C}$, however, indicate that the spin polarization itself and the DPF are not directly due to the bonding and antibonding states, nor to a peculiar effect of photoemission from a superconducting state, but they, rather, arise from a general phenomenon of interference in photoemission.

Within the present experimental capability, we have determined a sensibly larger EWS time delay in a cuprate superconductor when compared to the results on $\mathrm{Cu}(111)$. As a coarse explanation, it might be the case that the photoemission of strongly correlated electrons requires a longer sticking time before they may enter into quasifree particle states than those in a simple Fermi-liquid system, such as copper. 
Future experiments could shed light on the asymmetries of correlations going from the nodal to the antinodal direction in cuprate superconductors and the possible dependence of the time delay on temperature and stoichiometry. In order to make better quantitative comparisons and exhaustively study the dependence of spin polarization on several different parameters, however, a more systematic study is required, which will only become possible with the next generation of high-efficiency spin detectors [36,37] and with the development of a deeper theory of the relationship between spin polarization and time delay in photoemission from solid-state targets.

We gratefully acknowledge F. Carbone and M. Naamneh for fruitful discussions, and E. Giannini for providing us with the samples. This work was supported by the Swiss National Science Foundation Project No. PP00P2 144742/1.
[1] A. Damascelli, Phys. Scr. T109, 61 (2004).

[2] G. L. Bona, F. Meier, G. Schönhense, and H. C. Siegmann, Phys. Rev. Lett. 55, 1121 (1985).

[3] J. Kirschner, Polarized Electrons at Surfaces (Springer, Berlin, 1985).

[4] J. H. Dil, J. Phys.: Condens. Matter 21, 403001 (2009).

[5] T. Okuda and A. Kimura, J. Phys. Soc. Jpn. 82, 21002 (2013).

[6] E. E. Krasovskii, J. Phys.: Condens. Matter 27, 493001 (2015).

[7] D. Hsieh, Y. Xia, D. Qian, L. Wray, J. H. Dil, F. Meier, J. Osterwalder, L. Patthey, J. G. Checkelsky, N. P. Ong, A. V. Fedorov, H. Lin, A. Bansil, D. Grauer, Y. S. Hor, R. J. Cava, and M. Z. Hasan, Nature (London) 460, 1101 (2009).

[8] M. Z. Hasan and C. L. Kane, Rev. Mod. Phys. 82, 3045 (2010).

[9] S. V. Eremeev, G. Landolt, T. V. Menshchikova, B. Slomski, Y. M. Koroteev, Z. S. Aliev, M. B. Babanly, J. Henk, A. Ernst, L. Patthey, A. Eich, A. A. Khajetoorians, J. Hagemeister, O. Pietzsch, J. Wiebe, R. Wiesendanger, P. M. Echenique, S. S. Tsirkin, I. R. Amiraslanov, J. H. Dil, and E. V. Chulkov, Nat. Commun. 3, 635 (2012).

[10] G. Landolt, S. Schreyeck, S. V. Eremeev, B. Slomski, S. Muff, J. Osterwalder, E. V. Chulkov, C. Gould, G. Karczewski, K. Brunner, H. Buhmann, L. W. Molenkamp, and J. H. Dil, Phys. Rev. Lett. 112, 057601 (2014).

[11] U. Heinzmann and J. H. Dil, J. Phys.: Condens. Matter 24, 173001 (2012).

[12] U. Fano, Phys. Rev. 178, 131 (1969).

[13] D. T. Pierce and F. Meier, Phys. Rev. B 13, 5484 (1976).

[14] B. Sinkovic, L. H. Tjeng, N. B. Brookes, J. B. Goedkoop, R. Hesper, E. Pellegrin, F. M. F. de Groot, S. Altieri, S. L. Hulbert, E. Shekel, and G. A. Sawatzky, Phys. Rev. Lett. 79, 3510 (1997).

[15] C. N. Veenstra, Z. H. Zhu, M. Raichle, B. M. Ludbrook, A. Nicolaou, B. Slomski, G. Landolt, S. Kittaka, Y. Maeno, J. H. Dil, I. S. Elfimov, M. W. Haverkort, and A. Damascelli, Phys. Rev. Lett. 112, 127002 (2014).

[16] E. Razzoli, T. Jaouen, M.-L. Mottas, B. Hildebrand, G. Monney, A. Pisoni, S. Muff, M. Fanciulli, N. C. Plumb, V. A. Rogalev, V. N. Strocov, J. Mesot, M. Shi, J. H. Dil, H. Beck, and P. Aebi, Phys. Rev. Lett. 118, 086402 (2017).

[17] U. Heinzmann, J. Phys. B 13, 4353 (1980).

[18] J. Kessler, Polarized Electrons, 2nd ed. (Springer, Berlin, 1985).

[19] M. Fanciulli, H. Volfová, S. Muff, J. Braun, H. Ebert, J. Minár, U. Heinzmann, and J. H. Dil, Phys. Rev. Lett. 118, 067402 (2017).

[20] E. P. Wigner, Phys. Rev. 98, 145 (1955).
[21] A. L. Cavalieri, N. Muller, T. Uphues, V. S. Yakovlev, A. Baltuska, B. Horvath, B. Schmidt, L. Blumel, R. Holzwarth, S. Hendel, M. Drescher, U. Kleineberg, P. M. Echenique, R. Kienberger, F. Krausz, and U. Heinzmann, Nature (London) 449, 1029 (2007).

[22] R. Locher, L. Castiglioni, M. Lucchini, M. Greif, L. Gallmann, J. Osterwalder, M. Hengsberger, and U. Keller, Optica 2, 405 (2015).

[23] R. Kienberger (private communication).

[24] Y. D. Chuang, A. D. Gromko, A. Fedorov, Y. Aiura, K. Oka, Y. Ando, H. Eisaki, S. I. Uchida, and D. S. Dessau, Phys. Rev. Lett. 87, 117002 (2001).

[25] P. D. Johnson, A. V. Fedorov, and T. Valla, J. Electron Spectrosc. Relat. Phenom. 117-118, 153 (2001).

[26] A. Damascelli, Z. Hussain, and Z.-X. Shen, Rev. Mod. Phys. 75, 473 (2003).

[27] T. J. Reber, N. C. Plumb, Z. Sun, Y. Cao, Q. Wang, K. McElroy, H. Iwasawa, M. Arita, J. S. Wen, Z. J. Xu, G. Gu, Y. Yoshida, H. Eisaki, Y. Aiura, and D. S. Dessau, Nat. Phys. 8, 606 (2012).

[28] H. Anzai, A. Ino, M. Arita, H. Namatame, M. Taniguchi, M. Ishikado, K. Fujita, S. Ishida, and S. Uchida, Nat. Commun. 4, 1815 (2013).

[29] M. Hashimoto, I. M. Vishik, R.-H. He, T. P. Devereaux, and Z.-X. Shen, Nat. Phys. 10, 483 (2014).

[30] N. B. Brookes, G. Ghiringhelli, O. Tjernberg, L. H. Tjeng, T. Mizokawa, T. W. Li, and A. A. Menovsky, Phys. Rev. Lett. 87, 237003 (2001).

[31] M. Hoesch, T. Greber, V. N. Petrov, M. Muntwiler, M. Hengsberger, W. Auwaerter, and J. Osterwalder, J. Electron Spectrosc. Relat. Phenom. 124, 263 (2002).

[32] F. Meier, J. H. Dil, and J. Osterwalder, New J. Phys. 11, 125008 (2009).

[33] S. Weyeneth, T. Schneider, and E. Giannini, Phys. Rev. B 79, 214504 (2009).

[34] H. Ding, A. F. Bellman, J. C. Campuzano, M. Randeria, M. R. Norman, T. Yokoya, T. Takahashi, H. Katayama-Yoshida, T. Mochiku, K. Kadowaki, G. Jennings, and G. P. Brivio, Phys. Rev. Lett. 76, 1533 (1996).

[35] A. Piriou, E. Giannini, Y. Fasano, C. Senatore, and O. Fischer, Phys. Rev. B 81, 144517 (2010).

[36] V. N. Strocov, V. N. Petrov, and J. H. Dil, J. Synchrotron Radiat. 22, 708 (2015).

[37] C. Tusche, M. Ellguth, A. A. Ünal, C.-T. Chiang, A. Winkelmann, A. Krasyuk, M. Hahn, G. Schönhense, and J. Kirschner, Appl. Phys. Lett. 99, 32505 (2011). 